\title{
Diagnostic and Prognostic Value of Serum Tissue Polypeptide Antigen in Advanced Lung Cancer
}

\author{
Ahmed El Bastawisy'1*, Mahmoud El Azzouny², Gamal Mohamed², Ahmed Awad Allah², \\ Eman Behiry ${ }^{2}$ \\ ${ }^{1}$ Medical Oncology Department, National Cancer Institute, Cairo University, Cairo, Egypt \\ ${ }^{2}$ Clinical Pathology Department, Faculty of Medicine, Benha University, Benha, Egypt \\ Email: ${ }^{*}$ a s basta@hotmail.com
}

Received 3 February 2014; revised 28 February 2014; accepted 7 March 2014

Copyright (C) 2014 by authors and Scientific Research Publishing Inc.

This work is licensed under the Creative Commons Attribution International License (CC BY). http://creativecommons.org/licenses/by/4.0/

(c) (i) Open Access

\begin{abstract}
Lung cancer is a lethal malignancy, however, no serum marker is routinely recommended till now. Prospectively two groups of patients included: Group I: Patients with advanced lung cancer. Group II: patients with benign lung disease as control. Serum Tissue Polypeptide Antigen (TPA) levels were measured by ELISA technique before the first line chemotherapy. The TPA cutoff taken was $1800 \mathrm{pg} . / \mathrm{ml}$. End points were comparison of high TPA in cases and controls and correlation between high TPA and disease progression (PD), progression free survival (PFS) and overall survival (OS). 30 patients with advanced lung cancer (16 non-small and 14 small cell lung cancer) and 15 patients with benign lung disease were included and followed up during the period from October 2008 to October 2011 with median follow-up of 1.5 years. High TPA was found in $50 \%$ of lung cancer cases compared to $26 \%$ in controls $(p=0.014)$. High TPA was found in $64 \%$ of cases showing PD versus $36 \%$ normal TPA ( $p=0.08)$. 1 year PFS in high TPA was $32 \%$ versus $39 \%$ in normal TPA, $(p=0.2)$. 1 year OS in high TPA was $46 \%$ versus $73 \%$ in normal TPA $(p=0.6)$. Serum TPA is a potential marker for advanced lung cancer.
\end{abstract}

\section{Keywords}

Lung Cancer; Clinical Trials

\section{Introduction}

Lung cancer is a dismal disease with slight improvement in outcome over the past decade. Improvement in lung

*Corresponding author. 
cancer outcome is not only dependent on discovery of new drugs but also on identifying new diagnostic and prognostic markers. Perhaps one of the most important open issues in lung cancer is the in-availability of validated serum tumor markers which could assist in diagnosis and may spare the patient unnecessary interventional diagnostic procedures. Serum tumor markers can also serve a prognostic function when correlated to clinical outcome of the disease. Moreover it could open a new era for more drug able targets which may help to improve the outcome of advanced lung cancer. Several studies have investigated the usefulness of tumor markers in lung cancer mainly in staging, during post-therapeutic follow-up and for prognosis evaluation and even for early detection. Tissue polypeptide antigen (TPA) is a complex of polypeptide filaments of the cytokeoi7-iratins (CKs) 8, 18 and 19 and is produced during late S and G2 phases of the cell cycle. It is secreted into the circulation during and immediately after mitosis. TPA represents the most abundant CK pattern in malignant epithelial differentiation [1]. Several studies showed the value of TPA as a tumor marker although it was also elevated in nonmalignant conditions [2] [3]. However neither TPA nor any other serum tumor marker is recommended as a standard in lung cancer till now.

Aim of work: Primary end point was comparison of high TPA in cases and controls. Secondary endpoint was correlation between high TPA and disease progression (PD), progression free survival (PFS) and overall survival (OS) in advanced lung cancer patients.

\section{Patients and Methods}

This is a prospective case control study including two groups of patients: Group I: Patients with advanced lung cancer. Group II: patients with benign lung disease as control.

The study was conducted according to the Declaration of Helsinki and the guidelines for Good Clinical Practice. The local ethics committees approved the protocol.

\subsection{Inclusion Criteria}

Patient must have a pathological diagnosis of lung cancer, Stage IIIB \& Stage 4 lung cancer. Controls have benign lung disease diagnosed clinically. Patient is at least 18 years of age. Patient has a performance status of (ECOG Scale) $\leq 2$. Patient has adequate bone marrow function, (WBC count $\geq 3.0 \times 109 / \mathrm{L}$, ANC $\geq 1.5 \times 109 / \mathrm{L}$ platelet count $\geq 100 \times 109 / \mathrm{L}$, hemoglobin level $\geq 9$ g/L. Patient has adequate liver function; serum bilirubin $<1.5$ $\times$ ULN, ALT and AST levels < three times normal values; ALT and AST levels $<$ five times normal limits allowed in patients with known liver metastases. Patient has adequate Kidney function; plasma creatinine level $<1.5$ times normal value. Patients should have compliance, mental state and geographic proximity that allow adequate follow up and they have to provide written informed consent before any study-specific procedure.

\subsection{Exclusion Criteria}

Patient is pregnant or breastfeeding, patients with a "currently active" second malignancy, patient who is currently involved in another clinical trial.

\subsection{Treatment Plan}

Patients with NSCLC received the following chemotherapy regimen:

Gemcitabine: $1000 \mathrm{mg} / \mathrm{m}^{2}$, IV on 250 cc normal saline over $30 \mathrm{~min}$, D1, 8.

Cisplatin: $80 \mathrm{mg} / \mathrm{m}^{2}$ IV on $500 \mathrm{cc}$ normal saline over $1 \mathrm{hr}$, with standard hydration, D1.

Patients with SCLC received the following regimen:

Etoposide: $100 \mathrm{mg} / \mathrm{m}^{2}$, IV on 250 cc normal saline over $30 \mathrm{~min}$, D1 - 3.

Cisplatin: $80 \mathrm{mg} / \mathrm{m}^{2}$ D1, IV on 500 cc normal saline over $1 \mathrm{hr}$. with standard hydration.

Every 3 weeks, up to 6 cycles in responding patients, Patients with extensive stage SCLC showing any degree of clinical response received prophylactic cranial irradiation. Evaluation was done every 6 weeks.

\subsection{Study Assessment}

Pretreatment assessment included complete medical history and physical examination.

Further assessment conducted within 7 days before treatment included vital signs, performance status 
(ECOG), and Complete blood count with differential and full biochemical panel, including liver and renal function tests were performed and repeated before each treatment course.

Radiological evaluation includes computerized tomography (CT) scan of the chest and U/S Abdomen \& pelvis. Additional radiological imaging such as bone scan... was done if indicated. Imaging was repeated every 6 weeks.

Evaluation will be done according to RECIST.

\subsection{Post Treatment Evaluation}

Medical history and physical examination every 3 weeks, CBC and chemistry every 3 weeks, CT chest and upper abdomen every 6 weeks. Other investigations were done if indicated.

\subsection{Statistical Methods}

SPSS package (version 12.0) was used for data analysis. Mean and standard deviation were reported to describe quantitative data. The Chi-square and Fischer exact tests were used to evaluate the differences in the distribution of the variables. The Kaplan-Meier method was used to estimate the overall and progression free survival and the Log rank test to evaluate differences in survival among groups. P-value of 0.05 defined statistical significance.

\subsection{TPA Measurement Method}

ELISA Kit for Human Tissue Polypeptide Antigen (TPA) is supplied by Uscn, Life Science Inc.

REAGENT PREPARATION: All kit components and samples are brought to room temperature $\left(18^{\circ} \mathrm{C}\right.$ $25^{\circ} \mathrm{C}$ ) before use.

\section{1) Standard preparation:}

Standard is reconstituted with $1.0 \mathrm{ml}$ of standard diluent and kept for 10 minutes at room temperature, then shaked gently. The concentration of the standard in the stock solution is $10,000 \mathrm{pg}$. $/ \mathrm{ml}$. Then the stock solution is diluted to $5000 \mathrm{pg} . / \mathrm{ml}$ and serves as the highest standard (5000 pg. $/ \mathrm{ml})$. Seven tubes containing $0.5 \mathrm{ml}$ standard diluent are prepared and the diluted standard $(5000 \mathrm{pg} . / \mathrm{ml})$ is used to produce a double dilution series. Each tube is mixed thoroughly before the next transfer. Seven points of diluted standard are set up such as S1 (5000 pg./ml) S2 (2500 pg./ml), S3 (1250 pg./ml), S4 (625 pg./ml), S5 (312 pg./ml), S6 (156 pg./ml), S7 (78 pg./ml), and the last eppendorf tube with standard diluent is the blank as $0 \mathrm{pg} . / \mathrm{ml}$.

\section{2) Assay diluent $A$ and assay diluent $B$ preparation:}

Six $\mathrm{ml}$ of assay diluent A or B concentrate is diluted with $6 \mathrm{ml}$ of distilled water to prepare $12 \mathrm{ml}$ of assay diluent A or B.

\section{3) Detection reagent $A$ and detection reagent $B$ preparation:}

The stock detection A and detection B are briefly centrifuged before use. It is then diluted to the working concentration with working assay diluent A or B, respectively (1:100).

\section{4) Wash Solution preparation:}

Twenty $\mathrm{ml}$ of wash solution concentrate $(30 \mathrm{X})$ is diluted with $580 \mathrm{ml}$ of distilled water to prepare $600 \mathrm{ml}$ of wash solution.

\section{ASSAY PROCEDURE:}

Wells for diluted standard, blank and sample were determined (7 wells for standard and one well for blank). One hundred microliters $(100 \mathrm{~L})$ each of dilutions of standard, blank and samples were added into the appropriate wells and covered with the plate sealer and incubated for 2 hours at $37^{\circ} \mathrm{C}$. The liquid of each well was aspirated by pipettes. One hundred microliters $(100 \mathrm{~L})$ of detection reagent a working solution were added to each well, and incubated for 1 hour at $37^{\circ} \mathrm{C}$ after covering it with the plate sealer. The solution was aspirated and washed with $350 \mathrm{~L}$ of wash solution to each well using auto washer, and then sit for 1 - 2 minutes. The remaining liquid was removed from all wells completely by snapping the plate onto absorbent paper and repeated 3 times. One hundred microliters $(100 \mathrm{~L})$ of detection reagent $\mathrm{B}$ working solution was added to each well, and incubated for 30 minutes at $37^{\circ} \mathrm{C}$ after covering it with the plate sealer. The aspiration/ wash were repeated for five times. Ninety microliters $(90 \mathrm{~L})$ of substrate solution were added to each well and covered with a new plate sealer, then incubated for $15-25$ minutes at $37^{\circ} \mathrm{C}$, protected from light. The liquid turned blue by the addition of 
substrate solution. Fifty microliters (50 L) of stop solution were added to each well. The liquid turned yellow by the addition of stop solution. The liquid was mixed by tapping the side of the plate. Any drop of water and fingerprint on the bottom of the plate were removed. The micro plate was measured at an OD $450 \mathrm{~nm}$ immediately using a micro plate spectrophotometer reader.

\section{Results}

A total of 30 patients with advanced lung cancer (16 non-small and 14 small cell lung cancer ) and 15 patients with benign lung disease were included and followed up during the period from October 2008 to October 2011 with median follow up of one and half year.

\subsection{Patients' Characteristics}

Table 1 summarizes patients' characteristics with regard to age, sex, smoking history, histology, type and stage.

\subsection{Comparison between Cases and Control as Regards TPA}

Number of high TPA in cases is (15/30) 50\%, in control (4/15) 26\%. Number of normal TPA in cases is (15/30) $50 \%$, in control (11/15) 74\%. Cases of advanced lung cancer show statistical significant increase in TPA (tissue polypeptide antigen) than benign lung disease, p: 0.014 (Figure 1).

\subsection{Clinical Response}

There was a trend towards a statistically significant correlation between high TPA and progressive disease. High TPA was found in 64\% of cases showing PD versus 36\% normal TPA ( $p=0.08)$ Table 2.

\subsection{Survival}

\subsubsection{Progression Free Survival (PFS)}

There was no statistically significant correlation between high TPA and PFS. 1 year PFS in high TPA was 32\% versus 39\% in normal TPA ( $\mathrm{p}=0.2)$ Figure 2.

Table 1. Patients' characteristics.

\begin{tabular}{ccc}
\hline Character & Cases Number (\%) & Control Number (\%) \\
\hline AGE: & $24-75$ years (mean age = 59) & $32-7$ years (mean age $=45)$ \\
Sex: & & \\
Male & $23(76.6)$ & $9(60)$ \\
Female & $7(23.4)$ & $6(40)$ \\
Smoking: & & \\
YES & $21(70)$ & $8(53.3)$ \\
No & $9(30)$ & $7(46.7)$ \\
Histology: & & \\
Adenocarcinoma & $7(23.3)$ & - \\
Large cell carcinoma & $4(13.4)$ & \\
Squamous cell carcinoma & $5(16.6)$ & \\
Small cell carcinoma & $14(46.7)$ & $4(26.6)$ \\
Type: & & $6(40)$ \\
COPD & & $1(6.6)$ \\
Pleural effusion & & $1(6.6)$ \\
IPS & - & $2(6.6)$ \\
Empyema & & \\
Pneumonia & & - \\
hemoptysis & 19 & \\
Stage: & 11 & \\
III & & \\
IV & & \\
\hline
\end{tabular}


Table 2. Correlation between high, normal TPA and progression of disease.

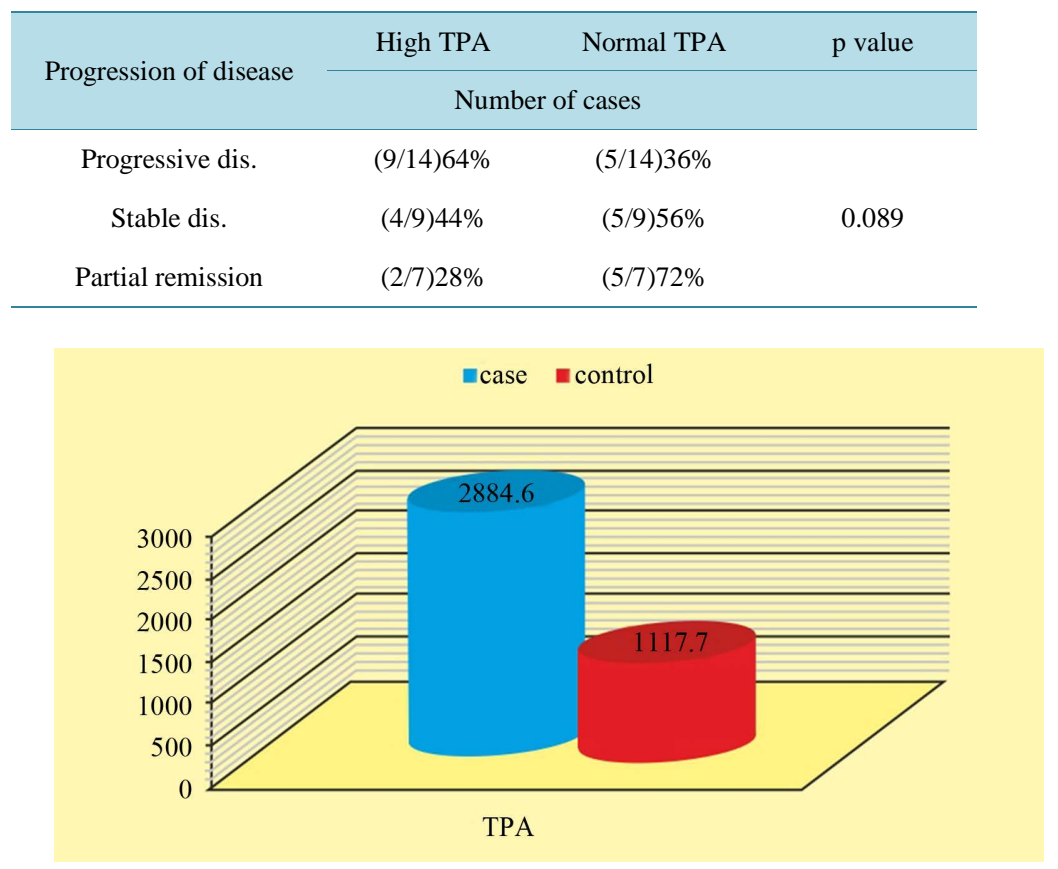

\section{Figure 1. Comparison between cases and control as regards TPA (pg./ml).}

Fig. (2): PEsurvival for TPA cases with high and normal level

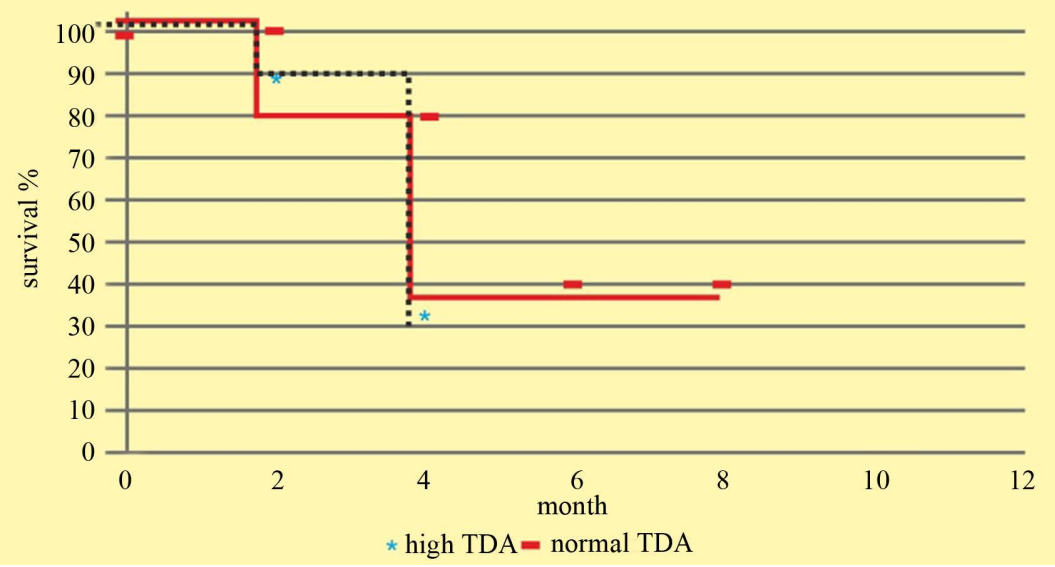

Figure 2. Correlation between high, normal TPA with progressive free survival.

\subsubsection{Overall Survival (OS)}

There was no statistically significant correlation between high TPA and OS.

1 year OS in high TPA was 46\% versus 73\% in normal TPA, $(p=0.6)$ Figure 3.

\section{Discussion}

Lung cancer is a dismal disease with poor outcome. Among methods to improve clinical outcome in lung cancer is to concentrate research not only on finding new drugs but also on methods of early diagnosis and monitoring of therapy [4]. It is unacceptable in the era of personalized medicine that we do not have serum tumor makers for diagnosis or follow up of lung cancer recommended in clinical practice. Thus, the present study was designed as an attempt to find out a rapid and reliable method for detection of advanced lung cancer rather than the ordinary invasive methods that had been used for diagnosis, again to define a prognostic value for this method. 


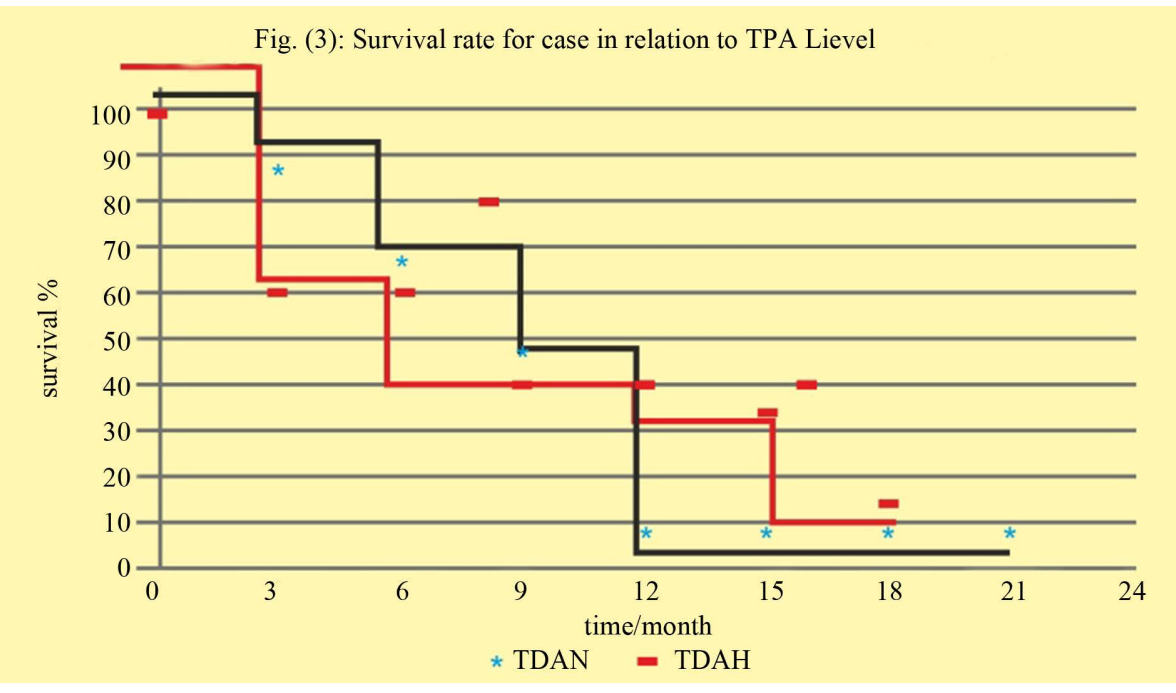

Figure 3. Correlation between high, normal TPA with overall survival.

This study included 45 patients.30 patients with histologically confirmed lung cancer and 15 with benign lung disease; they were classified into two groups.

Patients:

-Group I: Patients with histologically confirmed lung cancer as Non-small lung carcinoma and small cell lung carcinoma.

-Group II: patients with non-malignant lung disease.

In our study significant high TPA was found in $50 \%$ of lung cancer cases compared to $26 \%$ in controls ( $\mathrm{p}=$ 0.014). This is in concordance with studies done by Buccheri, 2001; Rapellino et al., 2003; Haga, 2003; Huang et al., 1997 [1] [4]-[6].

The present study showed a trend towards significant correlation between high TPA and progressive disease although not reaching statistical significance. Again the present study did not show significant correlation with PFS and OS in contrast to some previous studies which showed prognostic value for TPA like Foa et al., 2011; Kulpa et al., 2011 [7] [8]. This may be attributable to the relatively small sample size of the present study. This study is one step on the road which may ultimately lead to clinically validated serum tumor marker for lung cancer.

\section{Conclusion}

In our present study, it was concluded that serum TPA is a valuable tumor marker for advanced lung cancer carrying both diagnostic and a trend towards prognostic value. This study aims at re-attracting attention to focusing research on the important topic of identifying serum tumor markers for lung cancer with inclusion of larger number of patients.

\section{Acknowledgements}

This work was presented as poster in the 5th Asian pacific lung conference. Hong Kong, China 24, 25 August 2012.

\section{References}

[1] Buccheri, G. and Ferrigno, D. (2001) Lung Tumor Markers of Cytokeratin Origin: An Overview. Lung Cancer, 34, S65-S69.

[2] Lothgens, M. and Schlegel, G. (1983) Combined Use of Carcinoembryonic Antigen and Tissue Polypeptide Antigen in Oncologic Therapy and Surveillance. Cancer Detect Prevent, 6, 51.

[3] Holyoke, D. and Chu, T.M. (1997) Tissue Polypeptide Antigen. In: Herberman, R.B. and Mclntire, K.R., Eds., Immune-Diagnosis of Cancer, Dekker, New York, 513-521. 
[4] Rapellino, M., Pecchio, F., Baldi, S., Scappaticci, E. and Cavallo, A. (2003) Clinical Utility of Tissue Polypeptide Antigen Determination in Lung Cancer Management. Anticancer Research, 15, 1065-1070.

[5] Haga, E. (2003) Tissue Polypeptide Antigen in Serum and Tissue in Patients with Lung Cancer. Nihon Kyōbu Shikkan Gakkai zasshi, 28, 595-604.

[6] Huang, M.-S., Jong, S.-B., Tsai, M.-S., Lin, M.-S., Chong, I.-W., Lin, H.-C. and Hwang, J.-J. (1997) Comparison of Cytokeratin Fragment 19 (CYFRA 21-1), Tissue Polypeptide Antigen (TPA) and Carcinoembryonic Antigen (CEA) as Tumour Markers in Bronchogenic Carcinoma. Respiratory Medicine, 91, 135-142.

[7] Foa, P., Fornier, M., Miceli, R., Seregni, E., Santambrogio, L., Nosotti, M., Cataldo, I., Sala, M., Caldiera, S. and Bombardieri, E. (2011) Tumor Markers CEA, NSE, SCC, TPA and CYFRA 21.1 in Resectable Non-Small Cell Lung Cancer. Anticancer Research, 19, 3613-3618.

[8] Kulpa, J., Wójcik, E., Radkowski, A., Kolodziejski, L. and Stasik, Z. (2011) CYFRA 21-1, TPA-M, TPS, SCC-Ag and CEA in Patients with Squamous Cell Lung Cancer and in Chemical Industry Workers as a Reference Group. Anticancer Research, 20, 5035-5040. 